\title{
Rhodomycin analogues from Streptomyces purpurascens: isolation, characterization and biological activities
}

\author{
Sunita Holkar ${ }^{1 *}$, Deovrat Begde ${ }^{2}$, Nandita Nashikkar², Tukaram Kadam and Avinash Upadhyay ${ }^{2}$
}

\begin{abstract}
During a screening program for bioactive natural products, a potential Streptomyces sp was isolated from soil. On the basis of biochemical, cultural, physiological and 16S rRNA gene analysis, it was identified as Streptomyces purpurascens. The isolate was grown in liquid medium and the crude antibiotic complex was obtained by ethyl acetate extraction. Seven purified fractions were obtained by preparative Thin Layer Chromatography (TLC). Acid hydrolysis of each fraction and subsequent TLC led to the identification of aglycones and sugars indicating these compounds to be Rhodomycin and its analogues. The identity of these compounds was established on the basis of UV-visible and FT-IR spectra and comparison with published data. The compounds were active against Grampositive bacteria. Compound $\mathrm{E}$, identified as Rhodomycin B, was found to be the most potent compound with an MIC of $2 \mu \mathrm{g} / \mathrm{ml}$ against Bacillus subtilis. Compounds A and F identified as $a_{2}$-Rhodomycin II and Obelmycin respectively, and Compound E exhibited an $\mathrm{IC}_{50}$ of $8.8 \mathrm{\mu g} / \mathrm{ml}$ against HeLa cell line but no cytotoxicity was found against L929.
\end{abstract}

Keywords: Streptomyces purpurascens, Taxonomy, Rhodomycin, 165 rRNA, Bioactivity

\section{Introduction}

Anthracyclines, which include rhodomycins, are among the most widely studied natural products of Streptomyces $s p$. over the past quarter century (Vaněk et al. 1977). They attract attention because of their intense color that ranges from yellow and red to purple and blue. They belong to the group of aromatic polyketides where the basic structure is a cyclic polyketide backbone that shares the 7, 8, 9, 10-tetrahydrotetracene-5, 12-quinone structure. The diversity of these secondary metabolites is based on the structural differences in the aglycone and the different sugar residues attached (Additional file 1).

The anthracycline antibiotics have gathered attention of the chemists due to their outstanding antibacterial and antitumor activities (Hortobagyi 1997). Rhodomycin was the first anthracycline compound identified by H. Brockmann in 1963(Brockmann \& Bauer 1950). Since then many members of the

\footnotetext{
* Correspondence: sbundale@gmail.com

'Department of Microbiology, School of Life Sciences, Swami Ramanand Teerth Marathwada University (SRTMU), Nanded 431 401, Maharashtra, India Full list of author information is available at the end of the article
}

anthracycline family have been discovered with a broad spectrum of activity. These include daunorubicin, doxorubicin, idarubicin, epirubicin, zorubicin and aclacinomycin A, all of which are obtained from Streptomyces $s p$. The clinical use of these drugs, however has been hampered by a number of undesirable side effects, the most serious being the dose related cardiotoxicity (Jones 1993; Rahman et al. 2007). There is therefore, a great interest in related natural or synthetic compounds having improved therapeutic indices. Many efforts have been made to modify the anthracycline molecule with the objective of developing analogues with a wider spectrum of activity and reduced toxicity (Taatjes et al. 1996;Gianni et al. 2008). Also efforts have been directed towards obtaining hybrid anthracyclines with less toxicity. However, the complexity of the structure and biosynthetic pathway of anthracyclines have posed difficulties in these efforts (Jansson et al. 2003).

Soil is a natural reservoir for microorganisms, particularly the spore forming Streptomyces and their antimicrobial products. It is a known fact that Streptomyces are an 
unsurpassed source of bioactive materials. Although soils have been extensively screened by the pharmaceutical industry for about 50 years, only a small fraction of the surface of the globe has been sampled, and only a small fraction of Streptomyces taxa has been discovered. There is a strong circumstantial evidence that the discovery of previously unknown natural products occurs when novel organisms are examined in either established or new pharmacological screening programs (Takahashi \& Omura 2003). There are probably many unexplored anthracycline producing Streptomyces in soil which are yet to be discovered and may have an answer to this glaring problem.

With this intention, a Streptomyces $s p$. was isolated from soil and investigated for several bioactivities. It has been identified as Streptomyces purpurascens and deposited in the Microbial Type Culture Collection (MTCC), Chandigarh, India (http://mtcc.imtech.res.in/) with the accession number MTCC 8547. The red colored ethyl acetate cell extract of the isolate showed a high antimicrobial activity and the TLC delivered several red and orange zones. In the present study we report isolation, taxonomy of the isolate, its antimicrobial and anticancer activities, partial purification and characterization of the anthracyclines produced.

\section{Material and methods}

\section{Isolation of the microorganism}

In a systematic screening program for isolation of bioactive Streptomyces, soil samples from different locations in and around Nagpur, Maharashtra, India, were collected and stored at $-20^{\circ} \mathrm{C}$. The soil samples were pretreated by heating at $60^{\circ} \mathrm{C}$ for 10 minutes. $1 \mathrm{~g}$ each of the treated soil sample was serially diluted at 1:10 and 1:100 in sterile distilled water and plated on Starch-casein (SC) agar plates (Thakur et al. 2007). The SC agar plates were incubated for 14 days at $30^{\circ} \mathrm{C}$ and the resulting colonies were subcultured and maintained on Yeast extract-malt extract (YEME) agar (Ouhdouch et al. 2001;Smaoui et al. 2012).

Unless otherwise mentioned, all the chemicals and solvents were purchased from Merck and culture media from Hi-media laboratories, Mumbai.

\section{Identification of the isolate}

The morphological and cultural characteristics of the isolate were assessed in accordance with the method described by Shirling and Gottlieb (1966); Thakur et al. (2007)'. Cultural characteristics were observed on YEME agar (ISP2), Oatmeal agar (ISP3), Inorganic salt Starch agar (ISP4), and Glycerol Asparagine agar (ISP5) after 14 days of culturing at $28^{\circ} \mathrm{C}$. The morphological characteristics were assessed via light microscopy of 7-day old cultures grown on ISP4. Color of aerial mycelium was determined from mature, sporulating aerial mycelia of the Streptomyces colonies on SC agar media (Pridham et al. 1958).

The presence of soluble pigments and the melanoid pigment was investigated on (ISP 2) and peptone yeast extract iron agar (ISP 6).

The diagnostic isomers of diaminopimelic acid (DAP) and whole-organism sugars of the isolate were analyzed by the procedure developed by Becker et al.(1965) Utilization of various carbon and nitrogen sources was examined. Each source was added at a final concentration of $1 \%(w /$ v) and $0.1 \%(\mathrm{w} / \mathrm{v})$ respectively. Degradation of starch was determined according to Gordon et al. (1974). Growth in the presence of sodium chloride was determined according to Tresner et al. (1971) and temperature and $\mathrm{pH}$ requirements were assessed on ISP 2 medium.

\section{Molecular characterization of the isolate}

A confirmatory taxonomic identification was done by the nucleotide sequencing of the 16S rRNA gene. Genomic DNA extraction, amplification and sequencing of the $16 \mathrm{~S}$ rRNA gene were performed as described earlier (Mayilraj et al. 2006). The $16 \mathrm{~S}$ rRNA gene was amplified with primers 8-27f (5'-AGAGTTTGATCCTGGCTCAG-3') and 1500r (5'AGAAAGGAGGTGATCCAGCCA-3'). The amplified DNA fragment was separated on 1\% agarose gel, eluted from the gel and purified using QIAquick gel extraction kit (Qiagen). The purified PCR product was sequenced with 27f, 519r (5-GWATTACCGCGGCKGCTG-3), 1087r (5-CTCGTTGCGGGACTTAACCC-3), 530f (5-TTCGTG CCAGCAGCCGCGG-3), 945f (5-GGGCCCGCACAAGC GGTGG-3) and 1492r respectively (Escherichia coli numbering system). The rDNA sequence was determined by the dideoxy chain-termination method using the Big-Dye terminator kit using ABI 310 Genetic Analyzer (Applied Biosystems, USA). Almost complete sequence (1458 bp) of 16S rRNA of strain MTCC 8547 was determined and was compared with those of other closely related taxa retrieved from the GenBank database. A phylogenetic tree was constructed by Neighbour-Joining plot (Perrière \& Gouy 1996). A sequence similarity search was done using GenBank BLASTN (Altschul et al. 1997). Sequences of closely related taxa were retrieved; aligned using Clustal X programme (Thompson et al. 1997) and the alignment was manually corrected. For the Neighbour-joining analysis (Saitou \& Nei 1987), the distances between the sequences were calculated using Kimura's two-parameter model (Kimura 1980). Bootstrap analysis was performed to assess the confidence limits of the branching (Felsenstein 1985).

\section{Growth and antibiotic production studies}

Antibiotic production was studied in Potato dextrose broth (PDB) and antibiotic production medium, GS medium containing glucose $10 \mathrm{~g}$, soyabean meal $10 \mathrm{~g}$, $\mathrm{NaCl} 10 \mathrm{~g}$ and $\mathrm{CaCO}_{3} 1 \mathrm{~g}$ in 1 litre of water (Singh et al. 
2009). The $\mathrm{pH}$ of PDB was adjusted to 6.8 and that of GS to 7 before sterilization. Seed culture was prepared in $100 \mathrm{ml}$ Erlenmeyer flasks containing $25 \mathrm{ml}$ GS medium by inoculating a loopful from a slant culture and incubating at $28^{\circ} \mathrm{C}$ in a shaker incubator at $150 \mathrm{rpm}$ for 48 hours. Antibiotic production was observed using the same medium by inoculating $200 \mathrm{ml}$ medium with $2.5 \%(\mathrm{v} / \mathrm{v})$ of seed culture and cultivating under identical incubation conditions for 8 days.

\section{Effect of carbon and nitrogen}

The effect of various carbon and nitrogen sources was studied on growth as well as antibiotic production. Glucose soyabean meal medium was used as a standard medium. Carbon sources like starch, cellobiose, galactose, mannose, raffinose, rhamnose, lactose, maltose, fructose, sucrose, glycerol and xylose and nitrogen sources like casein, peptone, yeast extract, tryptone and jack bean meal were added at $1 \%$ concentration into the standard media with appropriate controls. After 10 days, each flask was harvested, the fermented broth as well as the cells were extracted with ethyl acetate, the extracts were dried in vacuum, resuspended in methanol to get a clear solution and tested for antibiotic activity against Bacillus subtilis. Growth was assessed by measuring cell mass.

\section{Isolation and purification of antimicrobial products}

The mycelial cake obtained from $200 \mathrm{ml}$ broth was extracted with $100 \mathrm{ml}$ acetone (Saito et al. 1995) and the spent broth $(200 \mathrm{ml})$ was extracted with $100 \mathrm{ml}$ chloroform. The acetone extract was concentrated to onefourth original volume and then reextracted with $25 \mathrm{ml}$ chloroform. The resulting chloroform extracts were concentrated and then excess of $60-80^{\circ} \mathrm{C}$ petroleum ether was added to obtain around $150 \mathrm{mg}$ of crude antibiotic complex (Eckardt et al. 1985).

The resulting dry broth and cell extracts were resuspended in a small volume of methanol and were tested for their antibacterial activity against B. subtilis by agar well diffusion method, but there was hardly any appreciable activity in the broth extract and hence it was not pursued further. All the other studies were carried out with only the cell extract. Preparative chromatography with Silica gel plate 60 F 254 (Merck, Germany, Cat \# 1.05554.0007) was used for the partial purification of antimicrobial products. The crude extract was spotted and developed in the solvent system Chloroform: methanol: 25\% aqueous $\mathrm{NH}_{3}$ (85:14:1) (Johdo et al. 1991a; Tobe et al. 1982). The developed plates were air dried overnight to remove all traces of solvents. Seven well separated bands were detected by observations of the color of the bands, which were orange to red. The TLC was repeated several times and the mean $\mathrm{R} f$ of the bands was calculated. The fractions were physically separated from each other by scraping the bands from the plates, extracting with methanol, concentrating the extracts and again subjecting each concentrate to TLC using the same solvent system, thereby obtaining each fraction in substantially pure form.

\section{Spectral studies}

The UV-visible absorption spectra (190 nm-1100 nm) of the TLC purified fractions were determined by using a double beam bio-spectrophotometer, (BL-198, Elico Ltd.). Futhermore, FT-IR spectrum of each active extract was detected using Shimadzu IR-470 plus. The spectra were scanned in the 400 to $4000 \mathrm{~cm}^{-1}$ range and plotted as intensity versus wave number (Augustine et al. 2005).

\section{Qualitative determination of aglycone and sugar residues} by TLC

To an aliquot of the purified fraction, $0.5 \mathrm{ml}$ of $0.1 \mathrm{~N}$ $\mathrm{HCl}$ was added and was heated at $85^{\circ} \mathrm{C}$ for 30 minutes in a water bath. The pigment aglycone thus obtained was extracted with $\mathrm{CHCl}_{3}$. The $\mathrm{CHCl}_{3}$ layer was evaporated to dryness and the pigment residue was chromatographed on a Silica gel plate 60 F 254 (Merck, Germany, Cat \# 1.05554.0007) using $\mathrm{CHCI}_{3}$ : $\mathrm{MeOH}(15: 1)$ mixture for chromatographic separation. The reference aglycones $\varepsilon$-rhodomycinone (RMN) (red), $\varepsilon$-isorhodomycinone (isoRMN) (purple), $\alpha$-citromycinone (CTN) (yellow), $\alpha 2-\mathrm{RMN}$ (orange), $\beta R M N$ (red), $\beta$-isoRMN (purple) and $\gamma$-isoRMN (purple) are documented to have $\mathrm{R} f$ values of $0.79,0.77$, $0.29,0.27,0.45,0.43$ and 0.59 , respectively (Johdo et al. 1991a). The aglycones were also determined by spot colors on the TLC plate.

Alternatively, the aqueous layer containing sugar components was neutralized by adding silver carbonate with a small amount of charcoal and centrifuged. The supernatant fluid was concentrated in vacuo and chromatographed on TLC Silica gel 60 plate F254 (Merck, Germany, Cat \# 1.05554.0007) using a BuOH- acetic acid $-\mathrm{H}_{2} \mathrm{O}(4: 1: 1)$ solvent system. The sugar spots were detected with p-anisaldehyde $-\mathrm{H}_{2} \mathrm{SO}_{4}$ (each 5\%) in $90 \% \mathrm{EtOH}$ spray reagent followed by heating at $90^{\circ}$ (Johdo et al. 1991a).

\section{Bioactivity of the fractions}

Each crude extract and purified fractions were tested against Bacillus subtilis, Staphylococcus aureus, Escherichia coli and Pseudomonas aeruginosa using agar well diffusion technique. $15 \mu \mathrm{l}$ of $1 \mathrm{mg} / \mathrm{ml}$ stock extracts were used for the tests. The diameter of the inhibition zones was determined after 24 hours of incubation at $37^{\circ} \mathrm{C}$. MIC of the bioactive fractions was determined using broth dilution technique (Wiegand et al. 2008). 


\section{MTT-based cytotoxicity assay}

The cytotoxicity of bioactive fractions on established cell lines like HeLa and L929 was determined in vitro as described by Mosmann (1983); Begde et al. (2010). The cell lines were purchased from NCCS, Pune, India. Freshly passaged HeLa cells were centrifuged and washed with HBSS. The cell count was adjusted to $2.5 \times 10^{5}$ cells $/ \mathrm{ml}$ by suspending the cells in fresh DMEM with 10\% FBS (Invitrogen). This cell suspension was then transferred to a 96-well TC plate for the assay and the cells were allowed to grow for $24 \mathrm{hrs}$ at $37^{\circ} \mathrm{C}$. The spent medium was discarded and the adherent cells were exposed to a concentration gradient of each purified fraction supplemented in the medium to a final concentration of $2.5,5,10,15,20,25,30$ and $35 \mu \mathrm{M}$ respectively. The plate was incubated at $37^{\circ} \mathrm{C}$ in a humidified environment with $5 \% \mathrm{CO}_{2}$ in air for $48 \mathrm{~h}$. After incubation the cells were washed and resuspended in fresh medium containing MTT (Himedia) and were further incubated for $3 \mathrm{hrs}$. The MTT formazan produced by the viable cells was extracted in DMSO (BIOGENE Reagents Inc., CA, USA) and quantified at $570 \mathrm{~nm}$ in the Bio-Rad ELISA plate reader.

\section{Results}

During a screening program for natural bioactive products, several microorganisms were isolated and their antimicrobial activity was studied. One of the cultures showing antibacterial activity was found to be promising and studied further.

\section{Taxonomy of the producer organism}

The morphological, physiological and cultural characteristics indicated that the soil isolate belonged to the genus Streptomyces. Streptomyces belong to the family Streptomycetaceae of the order Actinomycetales (Shirling \& Gottlieb 1966). The physiological characteristics and the cultural characteristics of the isolate are summarized in the Tables 1 and 2 respectively.

The comparison of the partial sequences (1458 bp) of $16 \mathrm{~S}$ rRNA of the isolate with those found in databases was done by BLASTN analysis. Result showed that query sequence of the isolate was best pair-wise aligned with 16S rRNA partial gene sequence of Streptomyces purpurascens strain: KN-6 (AB231806.1, score value 2751 bits) with similar sequence homology and identity of $100 \%$. The $\mathrm{N}-\mathrm{J}$ phylogenetic tree of the $16 \mathrm{~S}$ rDNA sequence data confirmed that the isolate was most closely related to S. purpurascens (Figure 1).

Out of $145816 \mathrm{~S}$ rRNA nucleotides of the isolate all were identical with those of $S$. purpurascens NBRC 13077, indicating that the sequence similarity of the $16 \mathrm{~S}$ rRNA of the two bacteria was $100 \%$. The culture has been deposited at Microbial Type Culture Collection,
Table 1 Physiological/Biochemical characteristics of Streptomyces purpurascens

\begin{tabular}{lc}
\hline Characteristics & S.purpurascens \\
\hline Temperature range of growth & $25-42^{\circ} \mathrm{C}$ \\
Urea hydrolysis & - \\
Starch hydrolysis & + \\
Caesin hydrolysis & - \\
Gelatin hydrolysis & + \\
Nitrate reduction & - \\
$\mathrm{H}_{2} S$ production & - \\
Citrate utilization & + \\
Catalase & + \\
Oxidase & - \\
Arabinose & + \\
Dextrose & + \\
Galactose & + \\
Fructose & + \\
Inositol & + \\
Raffinose & + \\
Rhamnose & + \\
Sorbitol & + \\
Salicin & + \\
Sucrose & + \\
Mylose & - \\
Lannitol & - \\
The table details the biochemical properties associated with the isolate & - \\
providing the biochemical guidelines towards identity of the organism. & - \\
\hline
\end{tabular}

Institute of Microbial Technology, Chandigarh, India (MTCC; http://mtcc.imtech.res.in/) as MTCC 8547.

\section{Effect of carbon and nitrogen sources}

Out of all the carbon sources tested for antibiotic production, Starch was found to be the best followed by cellobiose, raffinose, mannose and fructose. Of the nitrogen sources tested for antibiotic production, Yeast extract was the best followed by peptone and casein (Additional file 2: Figure S1).

\section{Physicochemical properties}

The purified fractions were named as $\mathbf{A}, \mathbf{B}, \mathbf{C}, \mathbf{D}, \mathbf{E}, \mathbf{F}$ and G. The stock solutions were preserved at $-20^{\circ} \mathrm{C}$.

The UV visible light absorption spectra of the purified fractions in methanol, against methanol blank exhibited characteristic peaks of anthracyclines in the range of $297 \mathrm{~nm}, 492-497,522-526$ and 557 -562 (except D) (Figure 2).

The FT-IR spectra (Figure 3) of all purified fractions indicated the presence of hydroxyl group (3400-3300 $\mathrm{cm}^{-1}$ ) 
Table 2 Cultural characteristics of S. purpurascens MTCC 8547

\begin{tabular}{|c|c|c|c|c|}
\hline \multirow[t]{2}{*}{ Media } & \multirow{2}{*}{$\begin{array}{l}\text { Color of mycelium } \\
\text { Orange }\end{array}$} & \multirow{2}{*}{$\begin{array}{l}\text { Spores } \\
\text { White + }\end{array}$} & \multirow{2}{*}{$\begin{array}{l}\text { Reverse side } \\
\text { Orange }\end{array}$} & Pigment \\
\hline & & & & Orange \\
\hline ISP2 & Orange & White +++ & Orange & Orange diffusible \\
\hline ISP3 & Orange & White + & Orange & Orange diffusible \\
\hline ISP4 & Orange & White +++ & Orange & Orange diffusible \\
\hline ISP5 & Violet & White +++ & Violet & Violet \\
\hline ISP6 & Yellow & - & Yellow & Brown \\
\hline
\end{tabular}




\begin{tabular}{|c|c|c|c|c|}
\hline ISP7 & Pink & - & Pink & No \\
\hline PDA & Orange & White+++ & Orange & Orange \\
\hline SDA & Orange & White+++ & Orange & Reddish orange \\
\hline
\end{tabular}

a ketonic group or ester carbonyl $\left(1740 \mathrm{~cm}^{-1}\right)$ and a hydrogen bonded carbonyl $\left(1600 \mathrm{~cm}^{-1}\right)$ which is characteristic of anthracyclines (Oki 1979). The properties of all fractions with their corresponding IR peaks are summarized in Table 3.

Qualitative determination of aglycone and sugars by TLC On acid hydrolysis, followed by TLC analysis, the purified fractions separated into red, orange and purple aglycones and corresponding sugar components. Except D, whose aglycone was found to be aklavinone, the aglycones of all the fractions were found to be rhodomycinone (RMN). Fractions $\mathbf{A}$ and $\mathbf{G}$ gave $\alpha 2-\mathrm{RMN}, \mathbf{B}$ and $\mathbf{E}$ gave $\beta$-RMN, $\mathbf{C}$ gave $\varepsilon$-RMN and $\mathbf{F}$ gave $\gamma$ isoRMN on hydrolysis. These aglycones were perihydroxy quinones was established by the color change of the red- orange bands to violet on staining with $0.5 \%$ ethanolic Magnesium acetate (Romanova et al. 1977). The sugars of all the fractions were found to be L-Rhodosamine and 2-deoxy-L- fucose by direct comparison with standard sugars on TLC. The details of the aglycones and sugars are summarized in Table 4.

\section{Identification of compounds}

Compound A appeared identical to $\alpha_{2}$-Rhodomycinone glycoside, probably $\alpha_{2}$-Rhodomycin II, which has been previously reported for S. purpurascens. Compound B was found to contain $\beta-R M N$ as the aglycone and L-Rhodosamine as the sugar, which indicated that it may be a Rhodomycin. The R $f$ and the UV-vis spectra of the aglycone of $\mathbf{B}$ matched that of $\beta$ - RMN. Also the UV-vis spectra of $\mathbf{B}$ was found to be similar to that of S-583 A-II (Shoji et al. 1968), which is reported to be a Rhodomycin B. Therefore it was considered that $\mathbf{B}$ is similar to Rhodomycin B. Compound C on acid hydrolysis yielded $\varepsilon$-rhodomycinone (RMN) and L-Rhodosamine and deoxy fucose as the sugars. Hence it was suspected to be a $\varepsilon$-rhodomycinone glycoside. Johdo et. al., have named such $\varepsilon$-rhodomycinone glycosides from $S$. violaceus as Epelmycins (Johdo et al. 1991b). The UV spectrum of C was found to match that of Epelmycin $\mathrm{C}$ with prominent peaks at $237 \mathrm{~nm}, 495 \mathrm{~nm}$ and $522 \mathrm{~nm}$. Also the $R f$ of purified $\mathbf{C}$ on silica gel TLC in solvent system $\mathrm{CHCl}_{3}$ : $\mathrm{MeOH}$ (8:1) was found to be 0.22 which coincided to the $R f$ of Epelmycin $\mathrm{C}$ in the same solvent system as reported by Johdo et. al. The FT-IR spectra (Figure 3) of $\mathbf{C}$ indicated the presence of an ester carbonyl $\left(1730 \mathrm{~cm}^{-1}\right)$ and a hydrogen bonded carbonyl $\left(1600 \mathrm{~cm}^{-1}\right)$ group which are characteristic of epelmycin anthracyclines (Johdo et al. 1991b). Also the absence of non-chelated carbonyl absorption $\left(1670 \mathrm{~cm}^{-1}\right)$ gave an indication that the aglycone could be $\varepsilon$-RMN rather than aklavinone (Doyle et al. 1979). Therefore this Rhodosaminosyl-decyfucosyl $\varepsilon$-rhodomycinone 


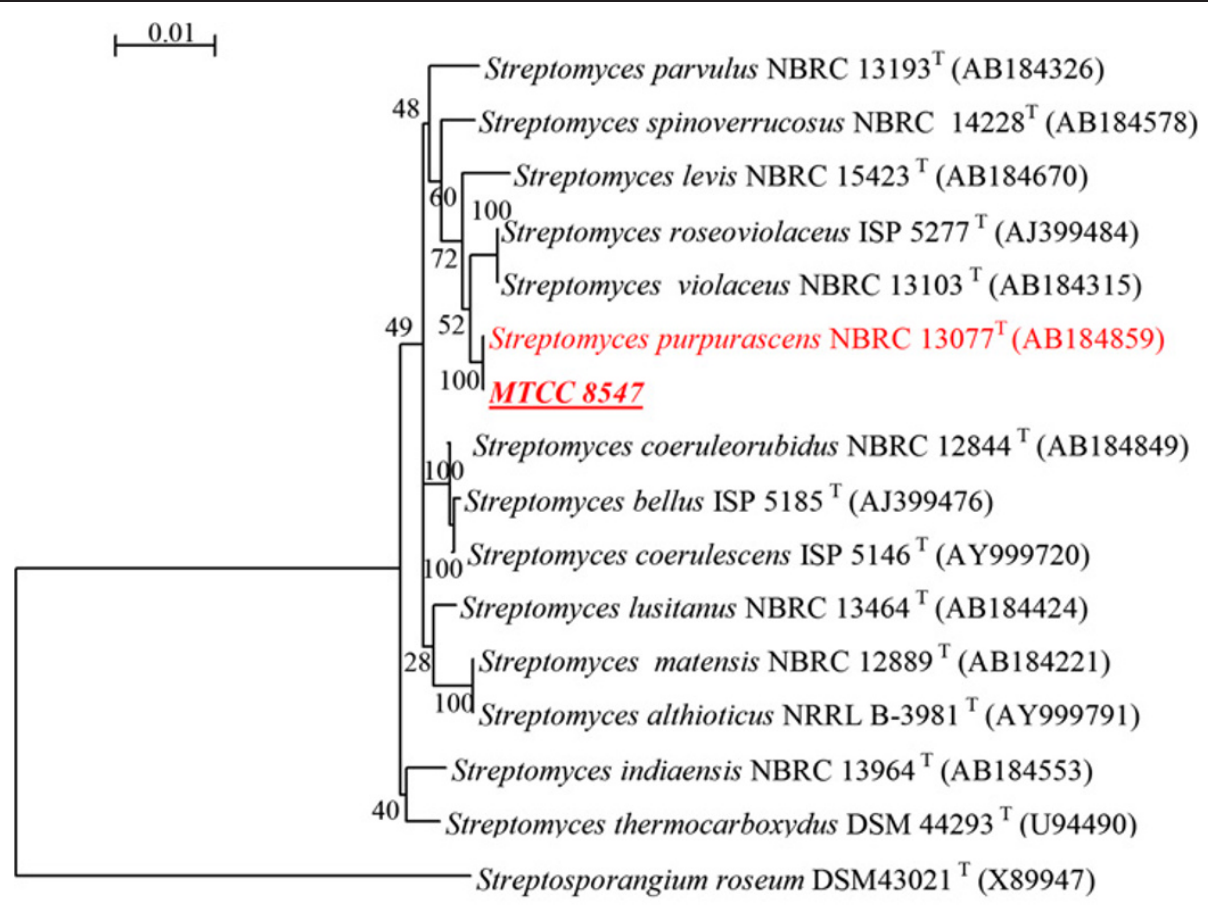

MTCC 8547 (Streptomyces purpurascens)1458 bp

CGCTGGCGGCGTGCTTAACACATGCAAGTCGAACGATGAACCACTTCGGTGGGGATTAGTGGCGAACGGGT GAGTAACACGTGGGCAATCTGCCCTGCACTCTGGGACAAGCCCTGGAAACGGGGTCTAATACCGGATATTG ACCATCTTGGGCATCTTTGATGGTGGAAAGCTCCGGCGGTGCAGGATGAGCCCGCGGCCTATCAGCTAGTT GGTGAGGTAATGGCTCACCAAGGCGACGACGGGTAGCCGGCCTGAGAGGGCGACCGGCCACACTGGGACTG AGACACGGCCCAGACTCCTACGGGAGGCAGCAGTGGGGAATATTGCACAATGGGCGAAAGCCTGATGCAGC GACGCCGCGTGAGGGATGACGGCCTTCGGGTTGTAAACCTCTTTCAGCAGGGAAGAAGCGAAAGTGACGGT ACCTGCAGAAGAAGCGCCGGCTAACTACGTGCCAGCAGCCGCGGTAATACGTAGGGCGCGAGCGTTGTCCG GAATTATTGGGCGTAAAGAGCTCGTAGGCGGCTTGTCACGTCGGTTGTGAAAGCCCGGGGCTTAACCCCGG GTCTGCAGTCGATACGGGCAGGCTAGAGTTCGGTAGGGGAGATCGGAATTCCTGGTGTAGCGGTGAAATGC GCAGATATCAGGAGGAACACCGGTGGCGAAGGCGGATCTCTGGGCCGATACTGACGCTGAGGAGCGAAAGC GTGGGGAGCGAACAGGATTAGATACCCTGGTAGTCCACGCCGTAAACGGTGGGCACTAGGTGTGGGCAACA TTCCACGTTGTCCGTGCCGCAGCTAACGCATTAAGTGCCCCGCCTGGGGAGTACGGCCGCAAGGCTAAAAC TCAAAGGAATTGACGGGGGCCCGCACAAGCGGCGGAGCATGTGGCTTAATTCGACGCAACGCGAAGAACCT TACCAAGGCTTGACATACACCGGAAACGTCTGGAGACAGGCGCCCCCTTGTGGTCGGTGTACAGGTGGTGC ATGGCTGTCGTCAGCTCGTGTCGTGAGATGTTGGGTTAAGTCCCGCAACGAGCGCAACCCTTGTCCCGTGT TGCCAGCAGGCCCTTGTGGTGCTGGGGACTCACGGGAGACCGCCGGGGTCAACTCGGAGGAAGGTGGGGAC GACGTCAAGTCATCATGCCCCTTATGTCTTGGGCTGCACACGTGCTACAATGGCCGGTACAATGAGCTGCG ATACCGCGAGGTGGAGCGAATCTCAAAAAGCCGGTCTCAGTTCGGATTGGGGTCTGCAACTCGACCCCATG AAGTCGGAGTCGCTAGTAATCGCAGATCAGCATTGCTGCGGTGAATACGTTCCCGGGCCTTGTACACACCG CCCGTCACGTCACGAAAGTCGGTAACACCCGAAGCCGGTGGCCCAACCCCTTGTGGGAGGGAGCTGTCGAA GGTGGGACTGGCGATTGGGACGAAGTCGTAACAAGGTA

//

Figure 1 Neighbour-joining tree based on 16S rRNA (1458 bases) sequences, showing the phylogenetic relationship between strain MTCC 8547 and other phylogenetic neighbours. Streptosporangium roseum DSM $43021^{\top}$ (X89947) was used as out group. Bootstrap values expressed as percentage of 100 replications. Bar represents 1\% sequence variation. Below is $1458 \mathrm{bp} 16 \mathrm{~S}$ rRNA gene sequence determined by the dideoxy chain-termination method using the Big-Dye terminator kit. This sequence was used to perform the sequence similarity search using GeneBank BLASTN based on which the phylogenetic tree was constructed by NJ plot.

could be an Epelmycin as reported by Johdo et. al., for Streptomyces violaceus. Compound D was yellow in color showing similarity to Aclacinomycin B. On hydrolysis, it yielded aklavinone and the sugars, Rhodosamine and deoxyfucose. $\mathbf{D}$ gave an $\mathrm{R} f$ of 0.68 in $\mathrm{CHCl}_{3}: \mathrm{MeOH}: 25 \%$ $\mathrm{aqNH}_{3}$ which is reported for aclacinomycins in the same solvent system. Its UV-vis spectra appeared similar to that of aclcinomycin B with an absorption maxima at $437 \mathrm{~nm}$. Also the aglycone of $\mathbf{D}$ gave a single yellow band with an
$\mathrm{R} f$ of 0.48 which coincided to the $\mathrm{R} f$ of standard alkavinone (Connors et al. 1990) under similar conditions. It also exhibited a violet fluorescence which is reported for aklavinone (Batel et al. 1990).

Compound $\mathbf{E}$ on acid hydrolysis yielded $\beta-R M N$ and the sugar L-Rhodosamine and even its FT-IR peaks (Table 3 \& Figure 3) appeared similar to rhodomycin. Thus it resembled rhodomycin which is reported for S. purpurascens. The band of compound F on TLC was 


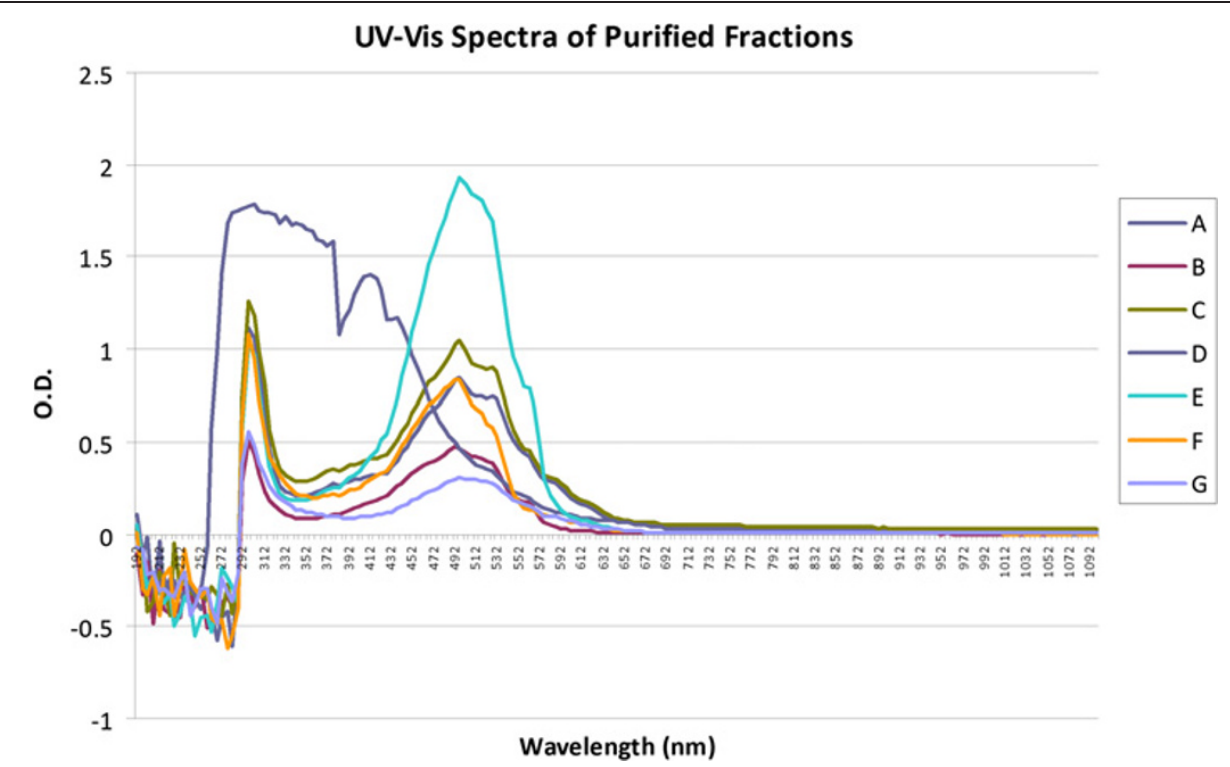

Figure 2 The UV visible absorption spectra of all the purified fractions, except $D$, exhibited characteristic peaks of anthracyclines in the range of $297 \mathrm{~nm}, \mathbf{4 9 2 -} 497 \mathrm{~nm}, \mathbf{5 2 2 -} 526 \mathrm{~nm}$ and $557-562 \mathrm{~nm}$. Absorption spectra for all the fractions were recorded in methanol, against methanol blank.

purple in color which on acid hydrolysis yielded the aglycone $\gamma$-iso RMN giving an indication that it may be similar to Obelmycin. The sugars detected in $\mathbf{F}$ were L-Rhodosamine and deoxyfucose. The fact that $\mathbf{F}$ contains $\gamma$-iso RMN and L-Rhodosamine and deoxyfucose led us to believe that $\mathbf{F}$ could be Obelmycin F. The belief was further strengthened by both having the same $\mathrm{R} f$ 0.48 in $\mathrm{CHCl}_{3}: \mathrm{MeOH}: \mathrm{NH}_{3}$ (80:10:1). Furthermore, the UV-visible spectra of $\mathbf{F}$ and Obelmycin F showed coinciding peaks at $297 \mathrm{~nm}, 492 \mathrm{~nm}$ and $527 \mathrm{~nm}$. And even the FT-IR spectra of $\mathbf{F}$ indicated the presence of hydrogen bonded carbonyl $\left(1600 \mathrm{~cm}^{-1}\right)$ which is characteristic of Obelmycins. But Obelmycin $\mathrm{F}$ is reported to contain L-Rhodinose along with L-Rhodosamine and 2-deoxy fucose. However, $\mathbf{F}$ did not show the presence of L-Rhodinose. $\mathbf{F}$ therefore was concluded to be similar to L-Rhodosaminosyl deoxyfucosyl $\gamma$-iso rhodomycinone. Compound $\mathbf{G}$ on acid hydrolysis yielded $\alpha 2$ rhodomycinone, hinting that it may be a $\alpha_{2}$-RMN glycoside, which have been designated as Alldimycins by Johdo et. al in $S$. violaceus. The $\mathrm{R} f$ of $\mathbf{G}$ in $\mathrm{CHCl}_{3}: \mathrm{MeOH}: \mathrm{NH}_{3}$ was 0.17 which exactly matched that of Alldimycin B (Johdo et al. 1991b). Also the UV-vis spectra showed a prominent peak at $497 \mathrm{~nm}$ similar to Alldimycins. Alldimycins have been reported to have only Rhodosamine as the sugar (Johdo et al. 1991b) whereas the hydrolysis product of $\mathbf{G}$ also yielded a spot for deoxy fucose on the chromatogram. Therefore, $\mathbf{G}$ perhaps was a new compound, resembling L- rhodosaminosyl, deoxyfucosyl $\alpha_{2}$-rhodomycinone which needs further confirmation.

\section{Bioactivity of purified compounds}

Antibacterial and antifungal activities were semiquantatively determined using agar well diffusion method where, Compound $\mathbf{E}$ was found to have the highest activity against B. subtilis and S. aureus followed by compound A. Compounds $\mathbf{C}$ and $\mathbf{F}$ demonstrated similar activities whereas compounds $\mathbf{D}, \mathbf{G}$ and $\mathbf{B}$ showed very weak activity (Figure 4). All the fractions failed to give any activity against Gram negative bacteria and Candida albicans.

The MIC of compounds $\mathbf{E}, \mathbf{A}$ and $\mathbf{F}$ was determined against $B$. subtilis by broth dilution method. The compound $\mathbf{E}$ was found to be very active with an MIC of $2 \mu \mathrm{g} / \mathrm{ml}$ whereas compounds $\mathbf{A}$ and $\mathbf{F}$ were comparatively less active with an MIC greater than $20 \mu \mathrm{g} / \mathrm{ml}$.

Furthermore, the compounds $\mathbf{E}, \mathbf{A}$ and $\mathbf{F}$ were found to be active against human cervical carcinoma HeLa cells with an $\mathrm{IC}_{50}$ of for $\mathrm{E} \sim 15 \mu \mathrm{M}$. However, in the tested range, these compounds did not demonstrate any appreciable cytotoxicity against mouse subcutaneous fibroblasts, L929 cells.

\section{Discussion}

In our screening program for anthracycline producers from soil, a Streptomyces $s p$. was isolated from soil. The isolate was identified as Streptomyces purpurascens by $16 \mathrm{~S}$ rRNA gene sequencing and deposited in Microbial Type Culture Collection (MTCC), Chandigarh, India (http:// mtcc.imtech.res.in/) with the accession number MTCC 8547. The isolate was found to produce rhodomycins which are reported for S. purpurascens. Apart 


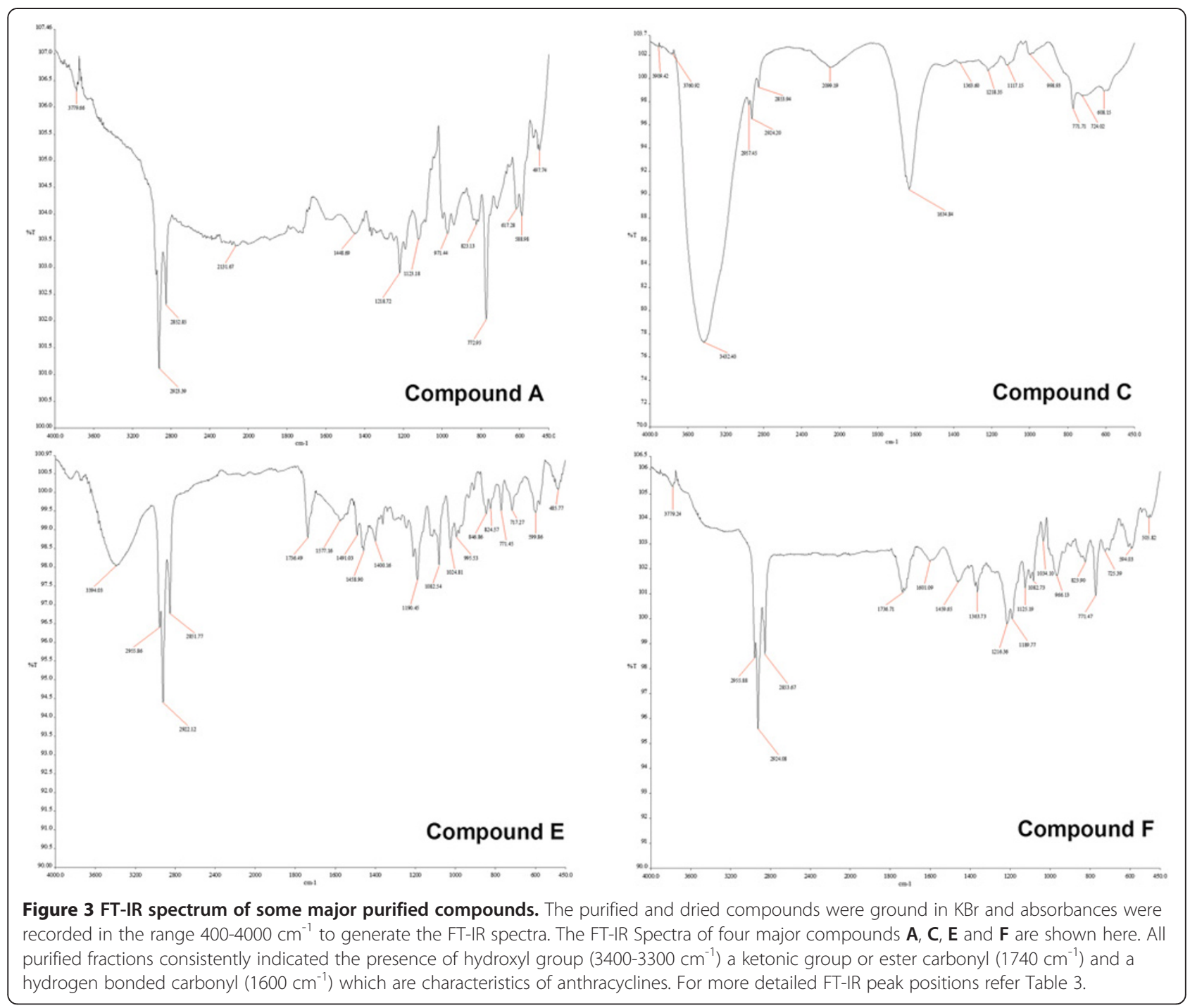

from this, some rhodomycin analogues which were not reported for S. purpurascens before were also obtained. In the present study at least one new rhodomycin analogue, was obtained from this isolate.

The isolate when grown in liquid culture produced red colored pigments extracellularly. Preliminary testing using the crude cell extract of the isolate showed it to be antibacterial against Gram positive bacteria. However, the crude extracts were not found to be active against Gram negative bacteria nor were they antifungal.

Studies on optimization of the growth medium indicated Starch as the best carbon source for growth as well as antibiotic production whereas yeast extract and peptone served as the best nitrogen source for antibiotic production and for growth respectively (For more details refer to Additional files). Therefore for all the further antibiotic production procedures the Starch-Yeast extract medium was preferred. Solvent extraction of the spent broth and subsequent purification using preparative TLC helped in obtaining the purified compounds. Seven purified compounds thus obtained were named A, B, C, D, E, F and G.

The UV visible spectra of all the fractions showed a peak in the 230-290 $\mathrm{nm}$ region indicating the presence of an aromatic ring in the structure. Also the FT-IR spectra of the compounds indicated the presence of hydroxyl groups, ketonic groups and hydrogen- bonded carbonyl groups which are characteristic of anthracyclines. Hence it was speculated that the isolate was an anthracycline producer. Identification of our isolate as $S$. purpurascens further strengthened the supposition since it is well known to produce anthracyclines belonging to the rhodomycin group (Brockmann \& Bauer 1950). On acid hydrolysis each compound yielded an 
Table 3 Tabulation of the physical and chemical properties of the compounds purified by preparative Thin Layer Chromatography

\begin{tabular}{|c|c|c|c|c|c|c|c|}
\hline Properties & A & B & $\mathrm{C}$ & D & $\mathrm{E}$ & $\mathrm{F}$ & G \\
\hline Color & Red & Orange & $\begin{array}{l}\text { Reddish } \\
\text { Orange }\end{array}$ & $\begin{array}{l}\text { Yellowish } \\
\text { Orange }\end{array}$ & Orange & Pink & Violet \\
\hline $\begin{array}{l}\text { Predicted Molecular } \\
\text { formula }\end{array}$ & ND & ND & $\mathrm{C}_{42} \mathrm{H}_{51} \mathrm{NO}_{16}$ & $\mathrm{C}_{42} \mathrm{H}_{53} \mathrm{NO}_{15}$ & $\mathrm{C}_{36} \mathrm{H}_{48} \mathrm{~N}_{2} \mathrm{O}_{12}$ & $\mathrm{C}_{40} \mathrm{H}_{53} \mathrm{NO}_{15}$ & $\mathrm{C}_{36} \mathrm{H}_{48} \mathrm{~N}_{2} \mathrm{O}_{12}$ \\
\hline Melting point $\left({ }^{\circ} \mathrm{C}\right)$ & ND & 184-190 & $175-178$ & $151-153$ & $185-190$ & $160-163$ & $134-137$ \\
\hline \multirow[t]{6}{*}{$U V \lambda_{\max }(n m)$} & $237(2.014)$ & 297(0.508), & 237(1.963), & 287(1.751), & 297(1.023), & 297(1.072), & 297(0.552), \\
\hline & $257(2.11)$ & 463(sh), & 257(sh), & 317 (1.74), & 497(1.923), & 492(0.834), & 377(0.101), \\
\hline & 284(1.957), & 492(0.472), & 495(0.483), & 375 (1.59), & 517(sh), & 527 (sh) & 497(0.304) \\
\hline & 492(0.614), & 526(sh), & $522(0.415)$ & $412(1.401)$ & $561(0.792)$ & & \\
\hline & $522(0.635)$ & $562(\mathrm{sh})$ & $562(\mathrm{sh})$ & 437 (1.172) & & & \\
\hline & $557(0.461)$ & & & & & & \\
\hline \multirow[t]{4}{*}{$\mathrm{IR}_{\max }(\mathrm{KBr})\left(\mathrm{cm}^{-1}\right)$} & 3780 & 3763 & 3760,3432 & ND & 3394,2922 & 3780,2924 & ND \\
\hline & 2924 & 2922 & 2924 & & 1736 & 1736 & \\
\hline & 2131 & 1723 & 1634,1363 & & 1577,1490, & 1600,1459 & \\
\hline & $\begin{array}{l}1450,1218 \\
1120,971,823\end{array}$ & $\begin{array}{l}1570,1458 \\
1192,1044,827\end{array}$ & $\begin{array}{l}1218,1117 \\
998\end{array}$ & & $\begin{array}{l}1458, \\
1190,1024,825\end{array}$ & $\begin{array}{l}1363,1189 \\
1034,823\end{array}$ & \\
\hline Rf value $^{a}$ & 0.88 & 0.80 & 0.79 & 0.68 & 0.62 & 0.48 & 0.17 \\
\hline
\end{tabular}

a the values were obtained on silica gel TLC, $\mathrm{CHCl}_{3}: \mathrm{MeOH}: 25 \% \mathrm{aqNH}_{3}$ (85:14:1); ND- Not Determined.

The table provides details about the UV-vis and FT-IR absorption peaks of respective purified compounds necessary for their identification.

aglycone and deoxy- sugars. The identity of the aglycone and sugars (by TLC) confirmed that these compounds might be rhodomycin and/or its anaologues (Johdo et al. 1991a).

Compound $\mathbf{A}$ appeared very similar to $\alpha_{2}$-Rhodomycin II and compounds $\mathbf{B}$ and $\mathbf{E}$ to that of Rhodomycins, which perhaps could be Rhodomycin A or B depending upon whether there are two rhodosamine moieties or one. All these compounds have been previously reported for S. purpurascens by Brockman (1955) and Shoji (1968; Arcamone 1984).

From our study, compound $\mathbf{C}$ appeared to be similar to an $\varepsilon$-RMN glycoside, Epelmycin and compound $\mathbf{F}$ resembled to an $\gamma$-isoRMN glycoside, Obelmycin. To the best of our knowledge, these compounds have not yet been reported for $S$. purpurascens. They have, however been reported for mutants of S. violaceus (Johdo et al. 1991c), which is very closely related and a phylogenetic

Table 4 A detailed description of sugar and aglycone substituent groups of all the isolated anthracyclines obtained from S. purpurascens

\begin{tabular}{|c|c|c|c|c|c|c|}
\hline \multirow[t]{2}{*}{ Fraction } & \multicolumn{2}{|l|}{ Aglycones } & \multirow{2}{*}{$\begin{array}{l}\text { Sugars } \\
\mathrm{Rf}^{\mathrm{b}}\end{array}$} & \multirow[b]{2}{*}{ Color } & \multirow[b]{2}{*}{ Sugar detected } & \multirow{2}{*}{$\begin{array}{l}\text { Compound } \\
\text { predicted }\end{array}$} \\
\hline & $\mathrm{Rf}^{\mathrm{a}}$ detected & Aglycone & & & & \\
\hline A & 0.27 & $a_{2}-R M N$ & 0.56 & grayish blue & 2-deoxy-L-fucose & $a_{2}$-Rhodomycin II \\
\hline \multirow[t]{2}{*}{ B } & 0.45 & $\beta$ RMN & 0.56 & grayish blue & L-Rhodosamine & Rhodomycin \\
\hline & & & & & 2-deoxy-L-fucose & \\
\hline C & 0.79 & $\varepsilon \mathrm{RMN}$ & 0.56 & grayish blue & 2-deoxy-L-fucose & Epelmycin B \\
\hline \multirow[t]{2}{*}{ D } & 0.67 & Aklavinone & 0.12 & sky blue & L-Rhodosamine & Aclacinomycin A \\
\hline & & & 0.56 & grayish blue & 2-deoxy-L-fucose & \\
\hline \multirow[t]{2}{*}{ E } & 0.45 & $\beta-R M N$ & 0.12 & sky blue & L-Rhodosamine & Rhodomycin $\mathrm{A}$ or $\mathrm{B}$ \\
\hline & & & 0.56 & grayish blue & 2-deoxy-L-fucose & \\
\hline \multirow[t]{2}{*}{$\mathrm{F}$} & 0.59 & $Y$-iso RMN & 0.12 & sky blue & L-Rhodosamine & Obelmycin \\
\hline & & & 0.56 & grayish blue & 2-deoxy-L-fucose & \\
\hline \multirow[t]{2}{*}{ G } & 0.27 & $a_{2}-R M N$ & 0.12 & sky blue & L-Rhodosamine & Alldimycin B \\
\hline & & & 0.56 & grayish blue & 2-deoxy-L-fucose & \\
\hline
\end{tabular}

a: $\mathrm{CHCl}_{3}: \mathrm{MeOH}$ (15:1), b: Butanol: acetic acid: water (4:1:1).

Identification of the sugars and aglycones were done by comparing their $\mathrm{R} f$ with that of the standards under similar TLC solvent conditions. 


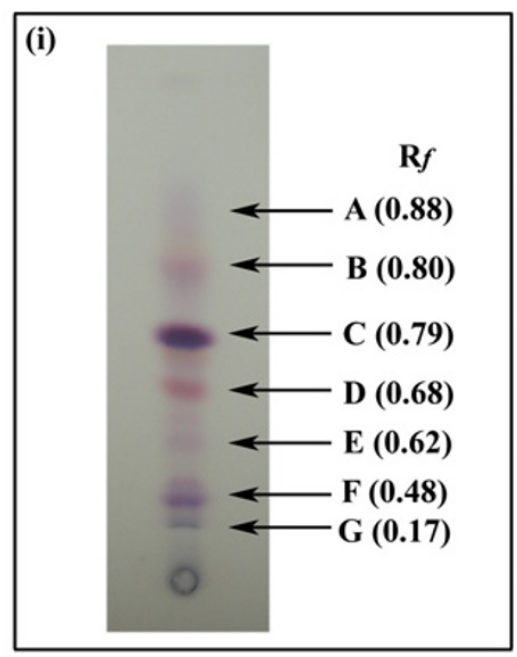

(ii)

\section{B. subtilis}

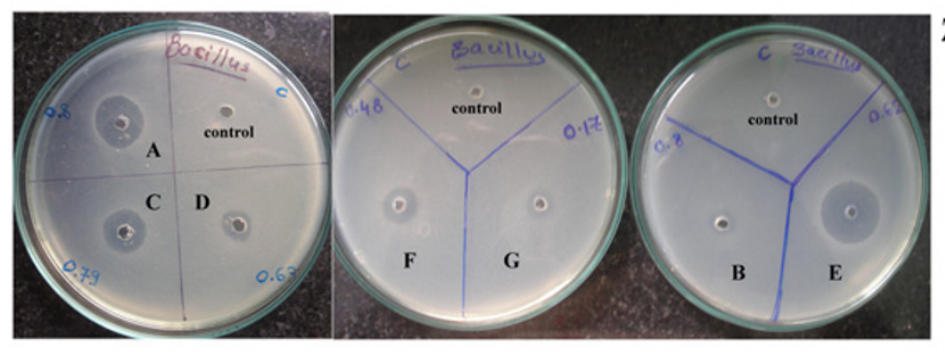

Zone of inhibition

( $\mathrm{c} \mathrm{m})$

A 0.6

B 0.1

C 0.4

D 0.3

E 0.8

F 0.4

G 0.2

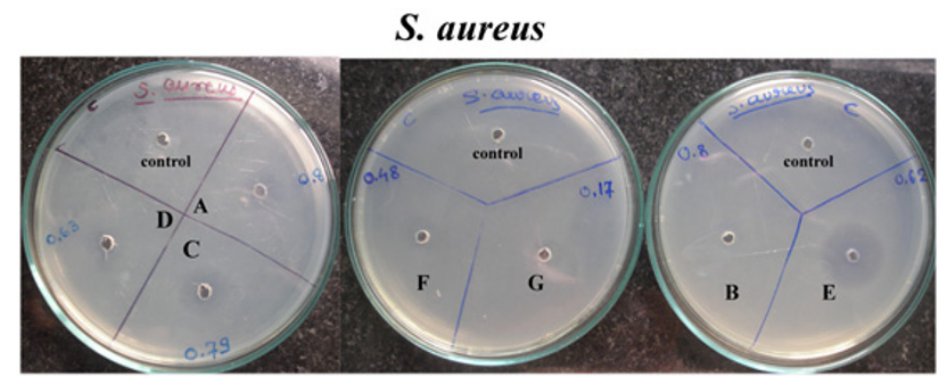

$\begin{array}{ll}\text { A } & 0.5 \\ \text { B } & 0.1 \\ \text { C } & 0.4 \\ \text { D } & 0.1 \\ \text { E } & 0.8 \\ \text { F } & 0.1 \\ \text { G } & 0.2\end{array}$

Figure 4 (i) Preparative TLC- For the partial purification of antimicrobial products preparative thin layer chromatography was performed. The crude extract got resolved into seven well separated colored bands when developed in the Chloroform: Methanol: 25\% aqueous $\mathrm{NH}_{3}$ (85:14:1) solvent system. According to the Rf value, each fraction was named from A to D. (ii) Antimicrobial activity of the isolated fractions against B. subtilis and $S$. aureus determined by agar well diffusion assay. Zone of inhibition associated with each fraction against respective test organisms is depicted.

neighbor of S. purpurascens as is evident from the Figure 1. The biosynthetic pathway for rhodomycin synthesis has been elucidated by Oki (1977), according to which during the synthesis of rhodomycin (a $\beta$-RMN glycoside), $\varepsilon$-RMN is an intermediate. This $\varepsilon$-RMN can be glycosylated by a glycosyl transferase to form $\varepsilon$-RMN glycosides, epelmycins. It has been suggested that biosynthetic conversion from $\varepsilon$-RMN to $\beta$-RMN takes place at glycoside level (Johdo et al. 1991b; Johdo et al. 1991c). Therefore formation of Epelmycins which are $\varepsilon$-RMN glycosides can be expected to occur in S. pupurascens too. Similarly, Compound D identified as an aklavinone glycoside,
Aclacinomycin A which is the main product in S. galilaeus has also not been reported for S. purpurascens. Aklavinone is the first aglycone during the synthesis of rhodomycin (Connors et al. 1990). Aklavinone can be glycosylated by a glycosyl transferase to form Aclacinomycin (Oki et al. 1979). Hence the possibility of obtaining aclacinomycins in the fermentation broth of S. purpurascens cannot be denied.

Compound G was identified as Rhodosaminyl-2Deoxyfucosyl- $\alpha_{2}$ Rhodomycinone. $\alpha_{2}$ RMN glycosides have been designated as Alldimycins by Johdo (Johdo et al. 1991d). All Alldimycins have been reported to 
contain only one sugar, Rhodosamine. Our studies consistently showed the presence of 2-deoxy fucose in the chromatogram of compound G. This leads us to believe that compound $\mathbf{G}$ may be a new rhodomycin analogue which has not been previously reported.

Antibacterial studies showed that compound $\mathbf{E}$ which appeared similar to Rhodomycin B was the most potent compound. Its MIC was found to be $2 \mu \mathrm{g} / \mathrm{ml}$ which was comparable to the reported value of $0.5-1 \mu \mathrm{g} / \mathrm{ml}$ (Shoji et al. 1968). However, the $\mathrm{IC}_{50}$ value, around $15 \mu \mathrm{M}$ $(\sim 8 \mu \mathrm{g} / \mathrm{ml})$ indicating much less toxicity than the reported value of $1 \mu \mathrm{g} / \mathrm{ml}$ (Saito et al. 1995).

Compound $\mathbf{A}$, identified as $\alpha_{2}$ Rhodomycin II and compound $\mathbf{F}$, identified as Obelmycin were found to be less potent. The MIC of both $\mathbf{A}$ and $\mathbf{F}$ was found to be $>20 \mu \mathrm{g} / \mathrm{ml}$ and $\mathrm{IC}_{50}$ was found to be around $15 \mu \mathrm{M}$ $(\sim 8 \mu \mathrm{g} / \mathrm{ml})$, which is much higher than the reported values. Compound $\mathbf{F}$ appeared similar to Obelmycins which are $\gamma$-isoRMN glycosides. These are reported to have low bioactivity because the sugar rhodosamine is bound at C-10 of the carbon skeleton (Johdo et al. 1991e). According to Vanek et al.(1977), the position of sugar moiety on the carbon skeleton is important. Anthracycline glycosides having C-7 sugar are more active than those having it at $\mathrm{C}-10$.

Thus this study resulted in the isolation of a rhodomycin producer from soil and its identification as Streptomyces purpurascens. The isolate was found to produce one novel Rhodomycin analogue and two known analogues being reported however, first time from S. purpurascens.

\section{Additional files}

Additional file 1: The general structure of the aglycone moiety for most commonly used anthracyclines. Common substituents are listed below the structure.

Additional file 2: Figure S1. Graphical representation of effect of carbon and nitrogen sources respectively on growth and antibiotic production of S. purpurascens. (A) Starch and (B) Yeast Extract appeared as best Carbon and Nitrogen sources respectively for the growth as well as for antibiotic production by S. purpurascens.

\section{Competing interests}

The authors declare that they have no competing interests.

\section{Authors' contribution}

SH carried out the microbiological and chromatography work, DB carried molecular genetic studies, NN participated in the sequence alignment, TK drafted the manuscript and AU critically revised the manuscript. All authors read and approved the final manuscript.

\section{Acknowledgement}

The authors would like to thank the Material Science Department of VNIT, Nagpur, Maharshtra, India for providing assistance in FT-IR analysis.

\section{Author details}

'Department of Microbiology, School of Life Sciences, Swami Ramanand Teerth Marathwada University (SRTMU), Nanded 431 401, Maharashtra, India.
${ }^{2}$ Hislop School of Biotechnology, Hislop College, Temple Road, Civil Lines, Nagpur, Maharashtra 440 001, India.

Received: 13 December 2012 Accepted: 22 February 2013

Published: 9 March 2013

\section{References}

Altschul SF et al (1997) Gapped BLAST and PSI-BLAST: a new generation of protein database search programs. Nucleic Acids Res 25(17):3389-3402

Arcamone F (1984) Antitumor anthracyclines: recent developments. Med Res Rev 4(2):153-188

Augustine SK, Bhavsar SP, Kapadnis BP (2005) A non-polyene antifungal antibiotic fromStreptomyces albidoflavus PU 23. J Biosci 30(2):201-211

Batel PL, Connors NC, Strohl WR (1990) Biosynthesis of anthracyclines: analysis of mutants of Streptomyces sp. strain C5 blocked in daunomycin biosynthesis. J Gen Microbiol 136(9):1877-1886

Becker B, Lechevalier MP, Lechevalier HA (1965) Chemical composition of cellwall preparations from strains of various form-genera of aerobic actinomycetes. Appl Microbiol 13(2):236-243

Begde D et al (2010) Immunomodulatory efficacy of nisin - a bacterial lantibiotic peptide. J Pept Sci 17(6):438-444. doi:10.1002/psc.1341

Brockmann H, Bauer K (1950) Rhodomycin, ein rotes antibioticum aus actinomyceten. Naturwissenschaften 37(21):492-493

Brockmann H, Patt P (1955) Iso-rhodomycin A, ein neues Antibioticum aus Streptomyces purpurascens, Rhodomycine, III. Mitteil.1); Antibiotica aus Actinomyceten, XXXII. Mitteil2). Chem Ber 88(9):1455-1468

Connors NC, Bartel PL, Strohl WR (1990) Biosynthesis of anthracydines: enzymic conversion of aklanonic acid to aklavinone and \{varepsilon\}-rhodomycinone by anthracycline-producing streptomycetes. J Gen Microbiol 136(9):1887-1894

Doyle TW et al (1979) Antitumor agents from the bohemic acid complex. 4. Structures of rudolphomycin, mimimycin, collinemycin, and alcindoromycin. J Am Chem Soc 101(23):7041-7049

Eckardt K et al (1985) Isolation and chemical structure of aklanonic acid, an early intermediate in the biosynthesis of anthracyclines. J Antibiot 38(8):1034-1039

Felsenstein J (1985) Confidence limits on phylogenies: an approach using the bootstrap. J Name Evol (US) J Vol 39(4):783-791, p. Medium: X; Size

Gianni L et al (2008) Anthracycline cardiotoxicity: from bench to bedside. J Clin Oncol 26(22):3777-3784

Gordan RE et al (1974) Nocardia coeliaca, nocardia autotrophica, and the nocardin strain. Int J Syst Bacteriol 24(1):54-63

Hortobagyi GN (1997) Anthracyclines in the treatment of cancer. An overview. Drugs 54(Suppl 4):1-7

Jansson A et al (2003) Crystal structure of aclacinomycin methylesterase with bound product analogues. J Biol Chem 278(40):39006-39013

Johdo O et al (1991a) Anthracycline metabolites from Streptomyces violaceus A262. I. Isolation of antibiotic-blocked mutants from Streptomyces violaceus A262. J Antibiot 44(10):1110-1120

Johdo O et al (1991b) Anthracycline metabolites from Streptomyces violaceus A262. II. New anthracycline epelmycins produced by a blocked mutant strain SU2-730. J Antibiot 44(10):1121-1129

Johdo O et al (1991c) Anthracycline metabolites from Streptomyces violaceus A262. I. Isolation of antibiotic-blocked mutants from Streptomyces violaceus A262. J Antibiot 44:1110-1120

Johdo O et al (1991d) Anthracycline metabolites from Streptomyces violaceus A262. V. New anthracycline alldimycin A: a minor component isolated from obelmycin beer. J Antibiot 44(10):1160

Johdo O et al (1991e) Anthracycline metabolites from Streptomyces violaceus A262. III. New anthracycline obelmycins produced by a variant strain SE2-2385. J Antibiot 44:1130-1140

Kimura M (1980) A simple method for estimating evolutionary rates of base substitutions through comparative studies of nucleotide sequences. J Mol Evol 16(2):111-120

Lown JW (1993) Anthracycline and anthraquinone anticancer agents: current status and recent developments. Pharmacology \&amp. Therapeutics 60(2):185-214

Mayilraj S et al (2006) Dietzia kunjamensis sp. nov., isolated from the Indian Himalayas. Int J Syst Evol Microbiol 56(7):1667-1671

Mosmann T (1983) Rapid colorimetric assay for cellular growth and survival: application to proliferation and cytotoxicity assays. J Immunol Methods 65(1-2):55-63

Oki T (1977) New anthracycline antibiotics. Jpn J Antibiot 30(Suppl):70-84 
Oki T (1979) Antitumor anthracycline antibiotics, aclacinomycin A and analogues. II.Structural determination. J Antibiot 32:801-819

Oki T et al (1979) Antitumor anthracycline antibiotics, aclacinomycin A and analogues. I. Taxonomy, production, isolation and physicochemical properties. J Antibiot 32(8):791-800

Ouhdouch Y, Barakate M, Finance C (2001) Actinomycetes of moroccan habitats: isolation and screening for antifungal activities. Eur J Soil Biol 37(2):69-74

Perrière G, Gouy M (1996) WWW-query: An on-line retrieval system for biological sequence banks. Biochimie 78(5):364-369

Pridham T, Hesseltine C, Benedict R (1958) A guide for the classification of streptomycetes according to selected groups. Placement of strains in morphological sections. Appl Microbiol 6(1):52

Rahman AM, Yusuf SW, Ewer MS (2007) Anthracycline-induced cardiotoxicity and the cardiac-sparing effect of liposomal formulation. Int J Nanomedicine 2 (4):567-583

Romanova AS, Patudin AV, Ban'kovskii Al (1977) Quinones of higher plants as possible therapeutic agents. Pharm Chem J 11(7):927-937

Saito S et al (1995) New rhodomycin analogs, SS-288A and SS-288B, produced by a Streptomyces violaceus A262 mutant. Biosci Biotechnol Biochem 59(1):135-137

Saitou N, Nei M (1987) The neighbor-joining method: a new method for reconstructing phylogenetic trees. Mol Biol Evol 4(4):406-425

Shirling EB, Gottlieb D (1966) Methods for characterization of Streptomyces species. Int J Syst Bacteriol 16(3):313-340, 10.1099/00207713-16-3-313

Shoji J et al (1968) Isolation of an antibiotic, S-583-B, related to rhodomycin A and B. J Antibiot 21(11):643

Singh V et al (2009) Optimization of actinomycin V production by Streptomyces triostinicus using artificial neural network and genetic algorithm. Appl Microbiol Biotechnol 82(2):379-385

Smaoui S et al (2012) Taxonomy, purification and chemical characterization of four bioactive compounds from new streptomyces sp. TN256 Strain. World J Microbiol Biotechnol 28(3):793-804

Taatjes DJ et al (1996) Alkylation of DNA by the anthracycline, antitumor drugs adriamycin and daunomycin. J Med Chem 39(21):4135-4138

Takahashi Y, Omura S (2003) Isolation of new actinomycete strains for the screening of new bioactive compounds. J Gen Appl Microbiol 49(3):141-154

Thakur D et al (2007) Isolation and screening of streptomyces in soil of protected forest areas from the states of assam and tripura, india, for antimicrobial metabolites. J Mycolog Méd J Med Mycolog 17(4):242-249

Thompson JD et al (1997) The CLUSTAL_X windows interface: flexible strategies for multiple sequence alignment aided by quality analysis tools. Nucleic Acids Res 25(24):4876-4882

Tobe $\mathrm{H}$ et al (1982) New anthracyclinone metabolites from two blocked mutants of Streptomyces galilaeus MA144-M1. J Antibiot 35(12):1641-1645

Tresner HD, Hayes JA (1971) Sodium chloride tolerance of terrestrial fungi. Appl Microbiol 22(2):210-213

Vaněk Z et al (1977) Anthracyclines. Folia Microbiol 22(2):139-159

Wiegand I, Hilpert K, Hancock REW (2008) Agar and broth dilution methods to determine the minimal inhibitory concentration (MIC) of antimicrobial substances. Nat Protocol 3(2):163-175

doi:10.1186/2193-1801-2-93

Cite this article as: Holkar et al:: Rhodomycin analogues from

Streptomyces purpurascens: isolation, characterization and biological activities. SpringerPlus 2013 2:93.

\section{Submit your manuscript to a SpringerOpen ${ }^{\circ}$ journal and benefit from:}

- Convenient online submission

- Rigorous peer review

- Immediate publication on acceptance

- Open access: articles freely available online

- High visibility within the field

- Retaining the copyright to your article

Submit your next manuscript at $>$ springeropen.com 